Voix et Images

voixetimages

\title{
Nelligan, Gauvreau, Ferron : perspectives critiques autour de la configuration de la folie et de l'écriture autobiographique. Présentation
}

\section{Christiane Kègle}

Volume 18, numéro 3 (54), printemps 1993

Littérature, folie, altérité

URI : https://id.erudit.org/iderudit/201042ar

DOI : https://doi.org/10.7202/201042ar

Aller au sommaire du numéro

\section{Éditeur(s)}

Université du Québec à Montréal

\section{ISSN}

0318-9201 (imprimé)

1705-933X (numérique)

Découvrir la revue

Citer ce document

Kègle, C. (1993). Nelligan, Gauvreau, Ferron : perspectives critiques autour de la configuration de la folie et de l'écriture autobiographique. Présentation. Voix et Images, 18(3), 448-452. https://doi.org/10.7202/201042ar 


\title{
Nelligan, Gauvreau, Ferron: perspectives critiques autour de la configuration de la folie et de l'écriture autobiographique. Présentation
}

\author{
Christiane Kègle, Université Laval
}

[...] on veut bien que l'écriture ait partie liée avec la folie, on ne veut même que ça, mais à condition que la folie reste en fin de compte une image, une figure; à condition, précisément, de ne pas avoir à la prendre à la lettre.

Présentée en guise d'exergue à mon propos, cette réflexion de Vincent Kaufmann ${ }^{1}$ introduit : d'emblée un parcours circulaire de l'écriture à la folie. Comme si le rapport de l'écrivain à l'imaginaire ne pouvait éviter l'écueil de ce lieu spéculaire incontournable où se tisse le rapport abyssal de soi à l'Autre. Vouloir endiguer les figures de la folie dans l'ordre tiers du langage, c'est aussi prendre le risque d'un effet de littéralisation de la lettre. Par un renversement dialectique, la folie finit alors par englober tout l'espace de représentation du discours, pour se résorber enfin dans l'aphanisis du sujet de l'énonciation.

Toute pratique d'écriture implique nécessairement une activité symbolique, aussi restreinte soit-elle. Lisez plutôt sous la plume du spécialiste des poèmes et textes d'asile ${ }^{2}$, comment Nelligan se fait un

1. Vincent Kaufmann, L'Équivoque épistolaire, Paris, Minuit, 1990, p. 194.

2. Soulignons au passage la publication des Euvres complètes d'Émile Nelligan, chez Fides en 1992. Tome 1: Poésies complètes, 1896-1941, édition critique établie par Réjean Robidoux et Paul Wyczynski. Tome 2: Poèmes et textes d'asile, 1900-1941, édition critique établie par Jacques Michon. Un certain critique à la courte vue n'a pas su rendre justice au travail magistral des auteurs, auxquels il faudra bien faire amende honorable ( $c f$. Spirale, mai 1992, p. 20). 
devoir inéluctable de répondre aux demandes d'autographes en $s$ 'inspirant des poésies de ses contemporains. Inapte à renouveler ses vers, c'est dans les préconstruits de la littérature que le poète devenu fou tisse l'ébauche d'une ouverture vers l'autre, cherche un fondement aussi réduit soit-il à ses relations au tiers. On ne saurait pourtant assimiler le tristement célèbre poète interné à la figure d'un plagiaire malgré lui. Car, ainsi que le souligne Jacques Michon, les quelques centaines de pages manuscrites rédigées par Nelligan dans les années trente ne comportent qu'une quantité négligeable de fausses signatures. Quelque image figée du poète "perdu dans ses songes" - que d'aucuns se sont plu à entretenir, comme pour créer un nécessaire effet de distanciation en regard de l'affreuse réalité asilaire - s'estompe devant l'analyse des transformations que Nelligan fait subir au langage. Anagrammatisations du prénom dans plusieurs variantes du répertoire des carnets; dissociations du nom et du prénom; fractures que les poèmes inédits font subir au nom du père; insertions du référent autobiographique dans le poème (par ces biographèmes qui témoignent souvent d'un sentiment d'appartenance à la culture irlandaise). À regarder de près la production de Nelligan après son internement, Jacques Michon retrace les dysfonctionnements de l'écriture: dérapages sémantiques, constructions verbales inusitées, mots inventés, rébus, devinettes, mots-valises, dérives des signifiants allant des phonèmes vers des ensembles plus vastes. Aussi, les poèmes et textes d'asile viennent-ils s'inscrire dans ce vaste corpus de la follittérature, dont Monique Plaza ${ }^{3}$ s'est efforçée ailleurs de définir les composantes.

Le texte fou ne laisse pas d'accuser un certain nombre d'écarts eu égard à la fonction référentielle du langage, voilà pourquoi la sémantique s'avère d'un apport précieux. D'autre part, la sémiotique peircéenne qui perçoit le signe comme participant de la vie collective, n'ayant d'existence que dans l'ordre du social — aux dires de Jean Fisette - demeure susceptible d'éclairer le rapport trouble de l'écrivain à son œuvre. A fortiori lorsque celle-ci s'inscrit dans un processus de mise en abyme du code de représentation, comme c'est le cas chez Gauvreau. Si la folie ressemble au signe dans la mesure où elle partage avec celui-ci son incomplétude et son instabilité, de poser le sémioticien, alors la question suivante s'avère probante: "En quoi une expérience de folie accède-t-elle à la signification?" Prenant soin de distinguer deux étapes successives et complémentaires chez Gauvreau - le surinvestissement de la poésie, suivi de la représentation des conditions

3. Monique Plaza, Écriture et Folie, Paris, Presses universitaires de France, coll. «Perspectives critiques:, 1986. 
de l'écriture - Jean Fisette cherche à démontrer comment, dans l'articulation de l'une à l'autre, s'élabore ce qu'il propose d'appeler la thérapie de la représentation. Car la pratique théâtrale chez Gauvreau, fortement investie par la dimension autobiographique, ne saurait se comprendre sans une référence rétroactive à l'influence des peintres automatistes, signataires avec Paul-Émile Borduas du Refus global. En pleine expérience poétique, dans la descente vertigineuse de l'imaginaire, Gauvreau se consacre à la déconstruction de toute forme de représentation. Reconduit au bord de l'abîme, il ne saurait remonter qu'en revendiquant par la suite, dans la position spéculaire du critique autoreprésenté sur la scène de son propre inconscient, "son droit inaliénable à participer à la vie collective avec sa folie". Et l'on sait par quelle descente aux enfers le poète dut payer son exploration des limites indéfinies du langage, lorsque ce dernier rompt avec la valeur prégrante du signe linguistique comme assise de la communication interpersonnelle.

Si les carnets de Nelligan ne comportent qu'un seul texte glossolalique (Premier), l'étude de Jean-Pierre Denis nous conduit par ailleurs vers les arcanes multiples du langage exploréen. Passant par un détour mallarméen, pour nous rappeler «la disparition élocutoire du poète, qui cède l'initiative aux mots", Jean-Pierre Denis s'attarde à la négativité qui empreint tout l'espace mortifère d'un travail d'écriture éminemment marqué par l'imago maternelle. Car les glossolalies chez Gauvreau ont partie liée avec a une langue barrée, emmurée vivante, une langue interdite. C'est en regard de la position singulière occupée par l'homme québécois dans la structure symbolique - plus précisément du défaut de la fonction paternelle - que la langue maternelle en vient à saturer dans la poésie gauvrienne le rapport du sujet à la jouissance et à la pulsion de mort. Selon Mallarmé encore, sont invoqués le lieu et les conditions de l'acte poétique par lesquels l'écrivain s'isole pour sculpter son propre tombeau. Il n'est pas étonnant, dès lors, de voir apparaître la figure prégnante de la bien-aimée, dont le deuil impossible revient comme un leitmotiv dans plusieurs œuvres. Au-delà d'une analyse des glossolalies, c'est à une réflexion plus profonde sur la quête de l'identité de l'homme québécois que nous convie le critique, suggérant en dernier lieu que la place laissée vide par le père absent aurait pu être occupée, dans le corpus de nos lettres québécoises, par le polygraphe Jacques Ferron.

Les figures de la folie sont d'ailleurs omniprésentes dans plusieurs textes de ce dernier, bien que l'appartenance de ceux-ci à un genre particulier demeure problématique. Si la forme épistolaire pratiquée par Ferron acquiert une plus grande portée que le seul récit du moi 
propre, le problème théorique que pose l'insertion constante de composantes autobiographiques dans son cuvre appelle une réflexion. Voilà pourquoi j'analyse le jeu des instances de discours dans l'ensemble intitulé "la configuration de la folie.", avant d'aborder la délicate question du transfert dans Le Pas de Gamelin. La troisième personne narrative, exclue de la corrélation de personnalité, constitue la pierre d'achoppement où vient buter incessamment l'écriture d'une œuvre.que l'écrivain considérait comme tout à fait ratée. C'est en référence au concept du "je-locuteur "que sont examinées la théorie du "truchement" et la problématique de la transposition littéraire, préoccupations qui reviennent par ailleurs incessamment dans la correspondance de l'écrivain avec le psychanalyste Julien Bigras. Par le truchement de Dieu se dessinent les figures christiques des folles de Gamelin qui finissent par se statufier dans le parcours métonymique qui les résume. Dans une visée heuristique, trois types de discours propres aux écrits ferroniens font ensuite l'objet d'une tentative de définition, soit les discours autobiographique, narratif et sociologique.

Pour clore le présent dossier de Voix et Images, Ginette Michaud propose un très long et dense article sur Ferron l'épistolier. Rappelant toute l'importance que revêt la correspondance chez cet écrivain prolifique, ayant soutenu "plusieurs correspondances très nourries et sur de fort longues périodes ", la spécialiste s'intéresse aux deux échanges épistolaires de Ferron avec le psychanalyste Julien Bigras et avec le professeur torontois John Grube. Non sans aborder auparavant la question de la correspondance privée comme lieu de réflexion, comme laboratoire d'écriture. C'est à la connaissance approfondie d'un Ferron "davantage pris par le jeu épistolaire qu'il ne saurait se l'avouer " que nous convie Ginette Michaud, dont la plume sait ménager quelque envolée sublime vers un lieu onirique universel. Qui ne se prendra à rêver devant l'évocation de la boîte-gigogne, cette image d'emboîtement spéculaire par excellence que constitue la grosse malle de pensionnaire dans laquelle, après les avoir déposées dans un grand sac, Ferron conservait toutes ses lettres? À l'étrange expérience du "ressouvenir *, rattachée aux lettres reçues en des temps anciens puis relues, le lecteur partagera avec l'écrivain et sa commentatrice le plaisir de ce mot ancien, issu du XVIII ${ }^{e}$ siècle. Par ailleurs, le terme d'échange épistolaire apparaît quelque peut inexact, de souligner Ginette Michaud, lorsque appliqué à la correspondance de Ferron avec Grube. Les lettres de l'écrivain à ce dernier, réunies et publiées chez Boréal en 1990, le sont en effet à sens unique. Et l'on regrettera que les relances du destinataire ne viennent éclairer les ombres, fantômes et fantoches déployés autour de la crise d'Octobre sur laquelle se trouve 
focalisé tout le jeu épistolaire. D'autant plus que la brève mais inquiétante étude de Georges Langlois, "Octobre en question ", publiée en annexe, n'est pas pour rassurer le citoyen hors de tout soupçon. Quoi qu'il en soit, et pour revenir à l'étude de Ginette Michaud, l'asile comme lieu d'enfermement n'est pas sans réactiver, tout au long de cette correspondance de Ferron, "des effets de réel liés à des épisodes anciens de captivité.. Or, dans l'échange avec le psychanalyste Julien Bigras, la position subjective de Ferron se révèle fort différente, de remarquer pertinemment la spécialiste, puisque l'écrivain semble constamment chercher «à sortir de la dualité clandestine et séductrice à laquelle Bigras veut le contraindre . Tout en poursuivant avec minutie sa lecture croisée des deux correspondances, lieux d'inscription des versants paternel et maternel du roman familial, Ginette Michaud souligne par ailleurs que "l'intérêt de ces lettres n'est pas seulement de servir d'appoint biographique, mais plus essentiellement d'attirer l'attention sur certains choix esthétiques déterminants faits par Ferron à cette époque». On lira donc avec un intérêt soutenu les remarques éclairantes de cette dernière sur un Ferron contraint de s'engager, à partir de 1975, sur la voie de l'autobiographie. Ce qui colore différemment la «nébuleuse des textes invoqués de manière redondante dans la correspondance, mais qui ne seront malheureusement jamais publiés du vivant de l'auteur. 